\title{
COMPOSIÇÃO FLORÍSTICA DA VEGETAÇÃO ARBÓREA DE UM REMANESCENTE DE CERRADÃO, PARAOPEBA, MG ${ }^{1}$
}

Priscila Bezerra de Souza ${ }^{2}$, João Augusto Alves ${ }^{3}$, Alexandre Francisco da Silva ${ }^{3}$ e Agostinho Lopes de Souza ${ }^{4}$

RESUMO - Este trabalho foi realizado na Floresta Nacional de Paraopeba - FLONA, Município de Paraopeba, Estado de Minas Gerais (19¹6'19"S e 44²4' 06"W), com os objetivos de conhecer a composição florística do estrato arbóreo de uma formação florestal (cerradão) e avaliar sua semelhança com outros levantamentos florísticos realizados em cerradões de Minas Gerais e São Paulo. Foram encontradas 78 espécies, pertencentes a 66 gêneros, representados em 31 famílias. As famílias mais representativas em números de espécies foram Fabaceae-Leguminosae (22), Myrtaceae (10) e Vochysiaceae (3). Floristicamente, os cerradões são mais próximos às formações florestais que aos Cerrados e poderiam, formalmente, ser classificados como um subtipo dessas formações florestais, visto que $83 \%$ das espécies são acessórias e apenas $17 \%$, peculiares.

Palavras-chave: Similaridade, cerradão e Floresta Nacional de Paraopeba.

\section{FLORISTIC COMPOSITION OF WOODY TREES OF A REMAINING CERRADÃO, PARAOPEBA, MINAS GERAIS}

\begin{abstract}
This work was carried out at the Floresta Nacional de Paraopeba - FLONA, in the municipality

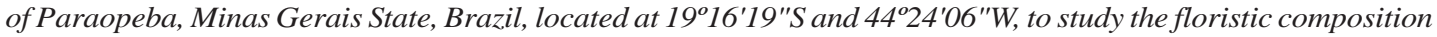
of the tree stratum of a forest formation (cerradão), and to evaluate the similarity with other floristic surveys conducted in cerradões of Minas Gerais and São Paulo. There have been found 78 species belonging to 66 genera representing 31 families. The most representative families in relation to number of species were Fabaceae-Leguminosae (22), Myrtaceae (10) and Vochysiaceae (3). Floristically the cerradões is closer to the forest formations than to cerrado and formally they could be classified as a subtype of those forest formations, because $83 \%$ of the species are accessory and just $17 \%$ peculiar.
\end{abstract}

Keywords: Similarity, cerradão and Floresta Nacional de Paraopeba.

\section{INTRODUÇÃO}

Segundo (COUTINHO, 1978), do ponto de vista fisionômico, os cerrados apresentam dois extremos: o cerradão, fitofisionomia na qual predomina o componente arbóreo-arbustivo, e o campo limpo, onde há predomínio do componente herbáceo-subarbustivo. As demais fitofisionomias encontradas - campo sujo, campo cerrado, cerrado sensu stricto - podem ser consideradas ecótonos entre o cerradão e o campo limpo.

Segundo Meira Neto (1991), as transições de cerrado sensu stricto para cerradão não são raras. Porém, é muito mais freqüente a transição de cerrado sensu stricto até campo cerrado, de maneira a formar um verdadeiro mosaico de fisionomias. A transição do cerrado sensu

\footnotetext{
${ }^{1}$ Recebido em 09.08.2007 e aceito para publicação em 19.05.2008.

${ }^{2}$ Programa de Pós-Graduação em Botânica da Universidade Federal de Viçosa(UFV), Viçosa-MG. E-mail: <pri_ufv@yahoo.com.br> .

${ }^{3}$ Departamento de Biologia Vegetal da UFV. E-mail: <j.meira@ufv.br>.

${ }^{4}$ Departamento de Engenharia Florestal da UFV. E-mail:<alsouza@ufv.br>.
} 
stricto para o cerradão ocorre de maneira suave, com aumento em tamanho dos indivíduos arbóreos, diminuição do número de arbustos, subarbustos e ervas, até que haja um estrato arbóreo, no qual é possível distinguirse uma camada contínua de copas que caracterizam um dossel. Pode-se afirmar que existem mais espécies no ambiente mais heterogêneo, diminuindo o número de espécies nas fisionomias menos complexas e, ou, com menores variações ambientais que o cerrado sensu stricto.

O cerradão apresenta dossel predominantemente contínuo e cobertura arbórea que pode oscilar de 50 a $90 \%$. A altura média do estrato arbóreo varia de 8 a $15 \mathrm{~m}$, proporcionando condições de luminosidade que favorecem a formação de estratos arbustivo e herbáceo diferenciados. Embora possam ser perenifólias, muitas espécies comuns ao Cerrado, como Caryocar brasiliense, Kielmeyera coriacea e Qualea grandiflora, apresentam caducifolia em determinados períodos na estação seca, nem sempre coincidentes com aqueles das populações do Cerrado (RIBEIRO et al., 1982).

Para (MARTINS, 1990), o levantamento florístico é um dos estudos iniciais para o conhecimento da flora de determinada área e implica produção de uma lista de espécies ali instaladas, que poderão contribuir para o estudo dos demais atributos da comunidade. Vários estudos da flora lenhosa já foram realizados em áreas de Cerrado (RIZZINI, 1971; RIBEIRO et al., 1985; MANTOVANI e MARTINS, 1993; COSTA ARAÚJO, 2001; WEISER e GODOY, 2001). Esses trabalhos evidenciaram a importância do bioma Cerrado, as suas fitofisionomias típicas e a elevada diversidade florística, sobretudo da flora arbustivo-arbórea, muito significativa e variada em relação aos outros estratos.

O conhecimento da composição florística em área demarcada abre perspectivas para o desenvolvimento de pesquisas relacionadas à fitossociologia, à fenologia e à dinâmica das populações ali instaladas. Do mesmo modo, o amplo conhecimento da flora do cerradão é um importante subsídio para o planejamento e implementação de áreas representativas dessa fitofisionomia, que devem ser priorizadas para conservação e manejo racional (FELFILI et al., 1993 e MENDONÇA et al., 1998).

Dessa forma o presente trabalho teve como objetivo determinar a composição florística de uma área de cerradão, na Floresta Nacional de Paraopeba; e avaliar sua semelhança com outros levantamentos florísticos realizados em cerradões de Minas Gerais e São Paulo.

\section{MATERIAL E MÉTODOS}

A Floresta Nacional de Paraopeba (FLONA), com área de 200 ha, localiza-se no município de Paraopeba - MG (19¹6'19"S e 4424'06"W), com altitude variando de $734 \mathrm{~m}$ ao sul a $761 \mathrm{~m}$, ao norte.

O clima da região foi caracterizado como subtropical úmido, com verão chuvoso e estação seca de abril a setembro, a precipitação média anual é de $1.236 \mathrm{~mm}$ e o déficit hídrico de 93 mm (THORNTHWAITE e MATHER, 1957; SILVA JÚNIOR, 1984).). Os solos foram classificados como Latossolo Vermelho-Escuro, Latossolo Vermelho-Amarelo e Latossolo-Amarelo (THIBAU et al., 1975; BALDUINO et al., 2005).

Foram instaladas 50 parcelas de 10 × 10 m, em cinco transectos paralelos de 10 x 100 m. A distribuição dos transectos foi feita sistematicamente a cada $20 \mathrm{~m}$ da borda. Nestas parcelas foram amostrados, todos os indivíduos com circunferência a 1,30 m do solo (CAP) maior ou igual a $10 \mathrm{~cm}$. Foram realizadas coletas botânicas de pelo menos um indivíduo de cada espécie por parcela. Aidentificação foi realizada através de comparações com material do Herbário VIC da Universidade Federal de Viçosa, literatura especializada e consultas a especialistas, quando necessário. Para atualização dos binômios específicos foram utilizados o índice de espécies Royal Botanic of Kew (1993) e o site do Missouri Botanical Garden (disponível em: http:/ /www.mobot.org/W3T/search/vast.html), no mês de julho de 2007. O sistema de classificação adotado foi o Botânica Sistemática, baseado em APG II (SOUZA e LORENZI, 2005). As espécies amostradas foram classificadas em acessórias e peculiares (RIZZINI, 1963).

A composição florística observada na FLONA foi comparada com listas de outros trabalhos realizados no Estado de Minas Gerais e São Paulo. Para o cálculo da similaridade foi utilizado o índice de Sørensen (Ss) (MUELLER-DOMBOIS e ELLENBERG 1974).

Para comparação florística entre as áreas selecionadas foram reunidos três trabalhos realizados em cerradões de São Paulo e dois de Minas Gerais, incluindo este. A interpretação dos dados se deu pelo método média de grupo (UPGMA) (SNEATH e SOKAL, 1973). Os dendrogramas foram produzidos por meio do programa FITOPAC (SHEPERD, 2002). 


\section{RESULTADOS E DISCUSSÃO}

Foram encontradas 78 espécies pertencentes a 66 gêneros, representando 31 famílias (Tabela 1). Entre as famílias, 30 pertencem à subclasse Magnoliopsida (dicotiledôneas), e somente uma, Arecaceae, a Liliopsida (monocotiledônea), estando representada por Acrocomia aculeata. Comparando estes dados com outros trabalhos realizados em cerradões de Minas Gerais e São Paulo, observou-se uma variação no número de espécies, sendo 78 em Uberlândia-MG (COSTA e ARAÚJO, 2001), 41 e 107 em Assis - SP (DURIGAN et al. 1997; 1999) e 51 em Águas de Santa Bárbara - SP Meira Neto (1991). Possivelmente o número de espécies poderia ser maior se não fosse à ação de "minhoqueiros", que revolvem as primeiras camadas do solo em busca dos minhocuçus (Oligochaeta), cortando, ao mesmo tempo, as plântulas e indivíduos jovens, alterando a dinâmica da comunidade (BALDUINO et al., 2005). Os poucos trabalhos publicados sobre a flora do cerradão indicam grandes mudanças na sua composição entre diferentes áreas dessa formação florestal.

O conjunto de espécies lenhosas, pertencentes à fitofisionomia de cerradão da (FLONA), apresentam relativa diversidade, contabilizando 1251 indivíduos. Os trabalhos de (RATTER et al., 1973; 1988) citaram valores sempre superiores de 100 árvores e arbustos grandes, para as diversas áreas da região core de cerrado. Essa diferença na diversidade também é verificada nos estudos realizados com cerradões (Meira Neto, 1991; DURIGAN et al., 1997; 199; COSTA e ARAÚJO, 2001).

As famílias mais ricas em espécies foram FabaceaeLeguminosae com (22 spp.), Myrtaceae (10 spp.), Sapindaceae (4 spp.), Vochysiaceae, Anacardiaceae, Rubiaceae, com (3 spp.), Annonaceae, Apocynaceae, Meliaceae, Malvaceae, Sapotaceae com (2 spp.), respectivamente. Dentre as Fabaceae, doze são FabaceaeFaboideae, seis Fabaceae-Caesalpinioideae e quatro Fabaceae-Mimosoideae. De acordo com a (Quadro 1), 21 famílias apresentaram apenas uma espécie. Comparando estes dados com outros trabalhos realizados em cerradões de Minas Gerais e São Paulo, observouse um número maior de famílias, sendo 41 em UberlândiaMG (COSTA e ARAÚJO, 2001) 37 e 53 em Assis - SP (DURIGAN et al., 1997; 1999) e 75 em Águas de Santa Bárbara - SP (Meira Neto, 1991). Porém foram menos representativas em riqueza de espécies.
A representatividade das famílias Fabaceae e Myrtaceae também foi verificada por (GOODLAND, 1969) no Triângulo Mineiro (MG), (FELFILI et al., 1993) na Chapada Pratinha (DF); (DURIGAN et al., 1999) na Estação Ecológica de Assis (SP); e (WEISER e GODOY, 2001) na ARIE cerrado Pé-de-Gigante (SP).

O destaque de Fabaceae em riqueza de espécies já era esperado, uma vez que essa família é de ampla distribuição no bioma cerrado, ocorrendo desde campo limpo a formações florestais e tem se destacado nos levantamentos realizados nessa formação Meira Neto (1991); (DURIGAN et al., 1997; COSTA e ARAÚJO, 2001; BALDUINO et al., 2005).

A vegetação da FLONA, em termos de semelhança florística, apresenta correlações com os cerradões de São Paulo e Minas Gerais. As espécies comuns nas áreas comparadas são Tapirira guianensis, Copaifera langsdorffii e Pera glabrata Meira Neto (1991); (DURIGAN et al., 1997; 1999; COSTAeARAÚJO, 2001). Deve-se ressaltar que tais espécies além de estarem presentes nos cerradões analisados, são também encontradas em matas mesófilas semidecíduas. De acordo com (RIZZINI, 1979; ARAÚJO e HARIDASAN, 1997a; ARAÚJO et al., 1997b; SOUZA et al., 2003) muitas espécies que ocorrem no cerradão são comuns em florestas mesófilas semidecíduas.

As espécies com maior número de indivíduos encontradas na FLONA de Paraopeba foram Myracrodruon urundeuva (123), Callisthene major (99) Cassia apoucouita (87), Rollinia sylvatica (77), Alibertia edulis (75), Siparuna guianensis (68), Aspidosperma subincanum (66) e Platypodium elegans (48). Essas oito espécies juntas contribuíram com 51,4\% do número total de espécies amostradas. Alguns taxons tidas como freqüente em quase todas as áreas de cerradão (RIZZINI e HERINGER, 1962 e RATTER et al., 1978), não estão presentes na FLONA de Paraopeba, dentre eles o Caryocar brasiliense, Emmotom nitens e a Lafoensia pacari. As espécies com maior densidade amostradas na FLONA de Paraopeba, podem ser encontradas em outras formações florestais ou savânicas. (FELFILI et al., 1994) afirmaram que na vegetação da chapada Pratinha (MG) não ocorre espécies exclusivas de cerradão, quer no estrato arbóreo, quer no estrato arbustivo.

Qualea grandiflora, distribuiu-se entre três cerradões, Assis 1999, Uberlândia e FLONA,

R. Árvore, Viçosa-MG, v.32, n.4, p.781-790, 2008 
corroborando as observações de (RATTER e DARGIE, 1992; RATTER et al., 1996), que, ao analisarem trabalhos de levantamentos florísticos em áreas de cerrado, observaram que essa espécie foi a mais amplamente distribuída, estando tanto em áreas de cerrado sensu stricto, cerradão e campo cerrado.

Quadro 1 - Listagem das espécies amostradas em 0,5 ha de formação florestal cerradão, na Floresta Nacional de Paraopeba (FLONA), em Paraopeba, MG, ordenadas alfabeticamente por famílias e subfamílias

Table 1 - List of sampled species in 0.5 ha of the forest formation (cerradão), in the National Forest - FLONA of Paraopeba - MG, in alphabetical order by families and subfamilies

\begin{tabular}{|c|c|c|}
\hline FAMÍLIAS-Subfamílias-Espécies & Peculiar & Acessória \\
\hline \multicolumn{3}{|l|}{1 ANACARDIACEAE } \\
\hline Astronium fraxinifolium Schott ex Spreng. & & $\mathrm{x}$ \\
\hline Myracrodruon urundeuva Allemao & & $\mathrm{X}$ \\
\hline Tapirira guianensis Aubl. & & $\mathrm{X}$ \\
\hline \multicolumn{3}{|l|}{2 ANNONACEAE } \\
\hline Rollinia sericea (R.E. Fr.) R.E. Fr. & & $\mathrm{X}$ \\
\hline Xylopia aromatica (Lam.) Mart. & $\mathrm{X}$ & \\
\hline \multicolumn{3}{|l|}{3 APOCYNACEAE } \\
\hline Aspidosperma olivaceum Müll. Arg. & & $\mathrm{x}$ \\
\hline Aspidosperma subincanum Mart. & & $\mathrm{x}$ \\
\hline \multicolumn{3}{|l|}{4 ARECACEAE } \\
\hline Acrocomia aculeata (Jacq.) Lodd. ex Mart. & & $\mathrm{x}$ \\
\hline \multicolumn{3}{|l|}{5 ASTERACEAE } \\
\hline Vernonia diffusa Less. & & $\mathrm{X}$ \\
\hline \multicolumn{3}{|l|}{6 BIGNONIACEAE } \\
\hline Tabebuia vellosoi Toledo & & $\mathrm{X}$ \\
\hline \multicolumn{3}{|l|}{7 BURSERSCEAE } \\
\hline Protium heptaphyllum (Aubl.) Marchand & & $\mathrm{X}$ \\
\hline \multicolumn{3}{|l|}{8 CANNABACEAE } \\
\hline Celtis iguanae (Jacq.) Sarg. & & $\mathrm{X}$ \\
\hline \multicolumn{3}{|l|}{9 CELASTRACEAE } \\
\hline Maytenus floribunda Reissek & & $\mathrm{x}$ \\
\hline \multicolumn{3}{|l|}{10 CHRYSOBALANACEAE } \\
\hline Hirtella hebeclada Moric. ex DC & & $\mathrm{X}$ \\
\hline \multicolumn{3}{|l|}{11 COMBRETACEAE } \\
\hline Terminalia argentea (Cambess.) Mart. & $\mathrm{x}$ & \\
\hline \multicolumn{3}{|l|}{12 EBENACEAE } \\
\hline Diospyros gaultheriaefolia Mart. \& Miq. & & $\mathrm{x}$ \\
\hline \multicolumn{3}{|l|}{13 EUPHORBIACEAE } \\
\hline Pera glabrata (Schott) Poepp. ex Baill. & & $\mathrm{x}$ \\
\hline \multicolumn{3}{|l|}{14 FABACEAE-Caesalpinioideae } \\
\hline Cassia apoucouita Aubl. & & $\mathrm{x}$ \\
\hline Hymenaea stigonocarpa Mart. ex Hayne & & $\mathrm{x}$ \\
\hline Peltophorum dubium (Spreng.) Taub. & $\mathrm{x}$ & \\
\hline Pterogyne nitens Tul. & & $\mathrm{x}$ \\
\hline Senna macranthera (DC. ex Collad.) H.S. Irwin \& Barneby & & $\mathrm{x}$ \\
\hline Bauhinia forficata Link & & $\mathrm{X}$ \\
\hline
\end{tabular}

R. Árvore, Viçosa-MG, v.32, n.4, p.781-790, 2008 
Quadro 1, cont.

Table 1, cont.

\begin{tabular}{|c|c|c|}
\hline FAMÍLIAS-Subfamílias-Espécies & Peculiar & Acessória \\
\hline \multicolumn{3}{|l|}{15 FABACEAE-Mimosoideae } \\
\hline Anadenanthera colubrina (Benth.) Brenan & & $\mathrm{X}$ \\
\hline Piptadenia gonoacantha (Mart.) J.F. Macbr. & & $\mathrm{X}$ \\
\hline Plathymenia foliolosa Benth. & & $\mathrm{X}$ \\
\hline Plathymenia reticulata Benth. & $\mathrm{X}$ & \\
\hline \multicolumn{3}{|l|}{16 FABACEAE-Faboideae } \\
\hline Amburana cearensis (Allemao) A.C. Sm. & & $\mathrm{x}$ \\
\hline Bowdichia virgilioides Kunth & & $\mathrm{X}$ \\
\hline Copaifera langsdorffii Desf. & & $\mathrm{X}$ \\
\hline Dipteryx alata Vogel & $\mathrm{X}$ & \\
\hline Machaerium hirtum (Vell.) Stellfeld & & $\mathrm{X}$ \\
\hline Machaerium nyctitans (Vell.) Benth. & & $\mathrm{X}$ \\
\hline Machaerium opacum Vogel & $\mathrm{X}$ & \\
\hline Machaerium scleroxylon Tul. & & $\mathrm{X}$ \\
\hline Machaerium stipitatum (DC.) Vogel & & $\mathrm{X}$ \\
\hline Platymiscium pubescens Micheli & & $\mathrm{X}$ \\
\hline Platypodium elegans Vogel & & $\mathrm{x}$ \\
\hline Swartzia macrostachya Benth. & & $\mathrm{X}$ \\
\hline \multicolumn{3}{|l|}{17 LAMIACEAE } \\
\hline Vitex megapotamica (Spreng.) Moldenke & & $\mathrm{x}$ \\
\hline \multicolumn{3}{|l|}{18 LAURACEAE } \\
\hline Endlicheria hirsuta (Schott) Nees & & $\mathrm{x}$ \\
\hline \multicolumn{3}{|l|}{19 MALPIGHIACEAE } \\
\hline Banisteriopsis anisandra (A. Juss.) B. Gates & & $\mathrm{X}$ \\
\hline \multicolumn{3}{|l|}{20 MALVACEAE } \\
\hline Apeiba tibourbou Aubl. & & $\mathrm{X}$ \\
\hline Ceiba speciosa St.-Hil. & & $\mathrm{X}$ \\
\hline Guazuma ulmifolia Lam. & & $\mathrm{x}$ \\
\hline Luehea divaricata Mart. & & $\mathrm{X}$ \\
\hline Zeyheria digitalis (Vell.) L.B. Sm. \& Sandwith & & $\mathrm{X}$ \\
\hline \multicolumn{3}{|l|}{21 MELASTOMATACEAE } \\
\hline Miconia langsdorffii Cogn. & & $\mathrm{x}$ \\
\hline \multicolumn{3}{|l|}{22 MELIACEAE } \\
\hline Trichilia hirta L. & & $\mathrm{X}$ \\
\hline Cedrela fissilis Vell. & & $\mathrm{X}$ \\
\hline \multicolumn{3}{|l|}{23 MORACEAE } \\
\hline Brosimum gaudichaudii Trécul & & $\mathrm{X}$ \\
\hline \multicolumn{3}{|l|}{24 MYRTACEAE } \\
\hline Campomanesia guazumifolia (Cambess.) O. Berg & & $\mathrm{x}$ \\
\hline Campomanesia velutina (Cambess.) O. Berg & & $\mathrm{X}$ \\
\hline Eugenia dysenterica DC. & $\mathrm{X}$ & \\
\hline Eugenia sulcata Spring ex Mart. & & $\mathrm{x}$ \\
\hline Gomidesia anacardiaefolia (Gardner) O. Berg & & $\mathrm{X}$ \\
\hline Myrcia lingua (O. Berg) Mattos \& D. Legrand & & $\mathrm{X}$ \\
\hline Myrcia rostrata DC. & $\mathrm{X}$ & \\
\hline Myrcia tomentosa (Aubl.) DC. & & $\mathrm{X}$ \\
\hline Pimenta pseudocaryophyllus (Gomes) Landrum & & $\mathrm{X}$ \\
\hline Psidium rufum DC. & & $\mathrm{X}$ \\
\hline
\end{tabular}


Quadro 1, cont.

Table 1, cont.

\begin{tabular}{|c|c|c|}
\hline FAMÍLIAS-Subfamílias-Espécies & Peculiar & Acessória \\
\hline \multicolumn{3}{|l|}{25 OCHNACEAE } \\
\hline Ouratea castaneifolia (DC.) Engl. & & $\mathrm{X}$ \\
\hline \multicolumn{3}{|l|}{26 PROTEACEAE } \\
\hline Roupala montana Aubl. & $\mathrm{X}$ & \\
\hline \multicolumn{3}{|l|}{27 RHAMNACEAE } \\
\hline Rhamnidium elaeocarpum Reissek & & $\mathrm{X}$ \\
\hline \multicolumn{3}{|l|}{28 RUBIACEAE } \\
\hline Alibertia edulis (Rich.) A. Rich. ex DC. & & $\mathrm{X}$ \\
\hline Guettarda viburnoides Cham. \& Schltdl. & & $\mathrm{X}$ \\
\hline Rudgea viburnoides (Cham.) Benth. & $\mathrm{X}$ & \\
\hline \multicolumn{3}{|l|}{29 SAPINDACEAE } \\
\hline Allophylus sericeus L. & & $\mathrm{X}$ \\
\hline Cupania vernalis Cambess. & & $\mathrm{x}$ \\
\hline Dilodendruon bipinatum Radl K. & & $\mathrm{X}$ \\
\hline Magonia pubescens A. St.-Hil. & $\mathrm{X}$ & \\
\hline \multicolumn{3}{|l|}{30 SAPOTACEAE } \\
\hline Pouteria tomentosa (Roxb.) Baehni & & $\mathrm{X}$ \\
\hline Pouteria venosa (Mart.) Baehni & & $\mathrm{X}$ \\
\hline \multicolumn{3}{|l|}{31 SIPARUNACEAE } \\
\hline Siparuna guianensis Aubl. & & $\mathrm{X}$ \\
\hline \multicolumn{3}{|l|}{32 STYRACACEAE } \\
\hline Styrax camporum Pohl & & $\mathrm{X}$ \\
\hline \multicolumn{3}{|l|}{33 VOCHYSIACEAE } \\
\hline Callisthene major Mart. & & $\mathrm{X}$ \\
\hline Qualea parviflora Mart. & $\mathrm{X}$ & \\
\hline Qualea grandiflora Mart. & $\mathrm{X}$ & \\
\hline
\end{tabular}

Se tomar em conta os critérios de espécies peculiares do Cerrado e acessórias segundo Rizzini (1963), podese dizer que, das 78 espécies identificadas (Quadro 1), apenas 13 (17\%) são peculiares e 65 (83\%), acessórias, o que leva a crer, segundo Rizzini (1963), Heringer et al. (1977) e Castro (1994), que, independentemente da sua origem florística, tanto o Cerrado quanto os outros biomas trocaram espécies ao longo do processo de adaptação da flora, sendo esse processo mais evidente nas áreas ecotonais.

(COSTAeARAÚJO, 2001) afirmaram que as espécies de formações florestais (matas) encontradas no cerradão parecem ocorrer em seu limite de distribuição, caracterizando o cerradão como uma possível área de transição entre as matas e o cerrado.

As relações florística, visualizadas por meio do dendrogramas gerado pela análise de agrupamento (UPGMA) a partir dos índices de similaridades de Søresen (Quadro 2), estão representadas na Figura 1. O dendrograma mostra graficamente que foram formados três grupos de amostra. O primeiro grupo é constituído pelo cerradão de Águas de Santa Bárbara e Assis 1997, sendo sua similaridade de aproximadamente 32\%, compartilhando 13 espécies (Quadro 3). 
Quadro 2 - Similaridade florística (Sørensen binário) entre cinco áreas de cerradão de Minas Gerais e São Paulo. 1 = Águas de Santa Bárbara Meira Neto (1991); 2 = Assis 1997 (DURIGAN et al., 1997); 3 = Assis 1999 (DURIGAN et al., 1999); 4 = Panga (COSTA e ARAUJO, 2001); e 5 = FLONA Paraopeba

Table 2 - Floristic similarity (binary Sørensen) of 5 for the areas of cerradão Minas Gerais and São Paulo. $1=$ Águas de Santa Bárbara Meira Neto (1991); 2 = Assis 1997 (DURIGAN et al., 1997); 3 = Assis 1999 (DURIGAN et al., 1999); 4 = Panga (COSTA e ARAUJO, 2001); 5 = (FLONA Paraopeba)

\begin{tabular}{|c|c|c|c|c|c|}
\hline 1 - Águas de Stª Bárbara & 1 & & & & \\
\hline 2 - Assis 1997 & 0,3200 & 1 & & & \\
\hline 3 - Assis 1999 & 0,2251 & 0,2559 & 1 & & \\
\hline 4 - Panga & 0,2930 & 0,2482 & 0,2761 & 1 & \\
\hline \multirow[t]{2}{*}{5 - Flona } & 0,1159 & 0,1525 & 0,1928 & 0,2400 & 1 \\
\hline & A & B & $\mathrm{C}$ & $\mathrm{D}$ & $\mathrm{E}$ \\
\hline
\end{tabular}

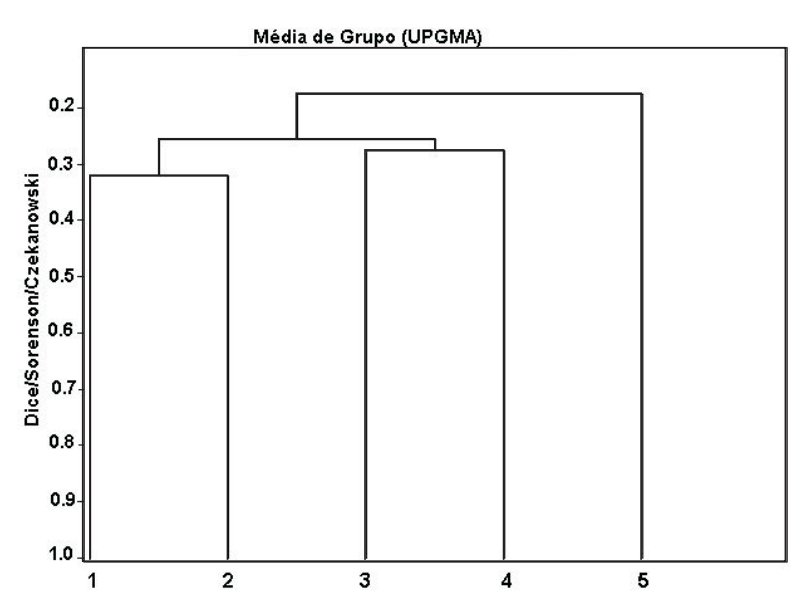

Figure 1 - Dendrograma da análise de agrupamentos por médias não-ponderadas (UPGMA) das similaridades florísticas (Sørensen), para as áreas de cerradão de Minas Gerais e São Paulo. 1 = Águas de Santa Bárbara (MEIRA NETO, 1991); 2 = Assis 1997 (DURIGAN et al., 1997); 3 = Assis 1999 (DURIGAN et al., 1999); 4 = Panga (COSTAARAUJO, 2001); e 5 = FLONA Paraopeba.

Figura 1 - Cluster anlysis dendrogram of unweighted pairgroup arithmetic average clustering method (UPGMA) of floristic similarity (binary Sørensen), for the areas of cerradão Minas Gerais and São Paulo. 1 = Águas de Santa Bárbara Meira Neto (1991); 2 = Assis 1997 (DURIGAN et al., 1997); 3 = Assis 1999 (DURIGAN et al., 1999); 4 = Panga (COSTA e ARAUJO, 2001); 5 = FLONA Paraopeba.

O segundo grupo (Assis 1999 e Panga) está reunido por uma similaridade de aproximadamente $27 \%$ e as espécies em comum e exclusivas estão no Quadro 3. O terceiro grupo, formado pela FLONA, é menos similar que os dois primeiros (Águas de Santa Bárbara, Assis 1997), (Assis 1999 e Panga), sugerindo-se que a posição geográfica, os fatores geológicos, as bacias hidrográficas, o clima e a altitude influenciaram os seus valores. Embora esta área esteja geograficamente mais distante e apresente menor similaridade, a mesma apresentou espécies comuns com outros cerradões analisados (Quadro 3).

A maior similaridade florística encontrada foi entre as áreas A e B (32\%) seguida por A e D (29\%) e a menor similaridade ocorreu entre as áreas A e E (12\%), além de se apresentarem mais distantes geograficamente. A explicação das maiores semelhanças apresentadas entre as áreas A, B e C provavelmente esteja relacionada com a proximidade geográfica e a matriz vegetacional.

Suponhamos que a semelhança entre o cerradão da FLONA de Paraopeba e o cerradão do Panga, seja devido a sua altitude que varia de 700 a $800 \mathrm{~m}$, por estarem sob ação de outros fatores ecológicos - talvez edáficos, climáticos - e geológicos, além de serem áreas de mosaico vegetacional grande heterogeneidade espacial de espécies.

Ao analisar as cinco áreas de cerradão distribuídas nos Estados de São Paulo e Minas Gerais (Meira Neto, 1991), (Águas de Santa Bárbara); (DURIGAN et al., 1997; 1999); (Assis); (COSTA e ARAUJO, 2001), (Uberlândia); e o presente trabalho (FLONA de Paraopeba), foi possível observar diferenças no número de espécies, supondo-se que tais diferenças, seja pelo estágio sucessional ou até mesmo o grau de antropização de cada área analisada.

R. Árvore, Viçosa-MG, v.32, n.4, p.781-790, 2008 
O resultado da análise de agrupamento mostra que áreas mais similares estão em estágio sucessional inicial (Águas de Santa Bárbara e Assis (97)) e as menos similares estão em estágio sucessional mais avançado, aumentando suas respectivas riquezas e se distanciando floristicamente por influência das Florestas Estacionais Semideciduais dessas regiões. É mais um agrupamento em favor de se poder considerar cerradões como formações florestais acima de certos valores de riqueza.

No cerradão estudado, as famílias mais ricas em número de espécies foram Fabaceae, Myrtaceae e Vochysiaceae. Dentre estas, Myrtaceae e Vochysiaceae se destacam nos Estados de Minas Gerais e São Paulo por apresentarem um elevado número de espécies, sendo responsáveis por grande parte da diversidade florestal.
Floristicamente, os cerradões são mais próximos às formações florestais que aos cerrados sensu stricto e poderiam formalmente ser classificados como um subtipo dessas formações florestais, visto que 83\% das espécies são acessórias e $17 \%$ peculiares.

A análise de agrupamento resultante do presente estudo pode representar diferenças na composição florística entre os cerradões dos Estados de Minas Gerais e São Paulo, diferenças estas que podem ser atribuídas a fatores ecológicos e geológicos. Constatouse que as espécies comuns a todas as áreas são espécies acessórias oriundas de formações florestais, parecendo ocorrer em seu limite de distribuição, caracterizando o cerradão como uma possível área de transição entre as matas e o cerrado.

Quadro 3 - Espécies exclusivas e de ligação do grupo (1), grupo (2) e grupo (3)

Table 3 - Some exclusive species and of connection of the group (1), group (2) and group (3)

\begin{tabular}{|c|}
\hline Grupo 1 \\
\hline Algumas espécies de ligação da área (A), Águas de Santa Bárbara e Assis 1997 \\
\hline $\begin{array}{l}\text { Anadenanthera falcata, Amaioua guianensis, Copaifera langsdorffii, Gochnatia polymorpha, Myrcia multiflora, Ocotea corymbosa, Styrax } \\
\text { camporum, Tapirira guianensis e Vochysia tucanorum. }\end{array}$ \\
\hline Algumas espécies de Águas de Santa Bárbara \\
\hline $\begin{array}{l}\text { Aspidosperma tomentosum, Bauhinia rufa, Campomanesia adamantium, Diospyros hispida, Erythroxylum tortuosum, Eugenia aurata, } \\
\text { Eugenia mansonii e Gochnatia barrosii. }\end{array}$ \\
\hline Algumas espécies exclusivas de Assis 97 \\
\hline Byrsonima ligustrifolia, Myrcia bella, Myrcia fallax, Protium widgrenii, Pseudolmedia laevigata e Trichilia pallida. \\
\hline Grupo 2 \\
\hline Algumas espécies de ligação da área (B), Assis 1999 e Panga \\
\hline $\begin{array}{l}\text { Acosmium subelegans, Agonandra brasiliensis, Annona crassiflora, Austroplenckia populnea, Bowdichia virgilioides, Byrsonima } \\
\text { coccolobifolia, Casearia sylvestris, Caryocar brasiliense, Coccoloba mollis, Copaifera langsdorffii e Couepia grandiflora. }\end{array}$ \\
\hline Algumas espécies exclusivas de Assis 1999 \\
\hline $\begin{array}{l}\text { Actinostemum conceptionis, Aeschynomene selloi, Alchornea triplinervia, Allophylus edulis, Ambrosia polystachya, Anadenanthera } \\
\text { colubrina, Andira inermis, Annona cacans, Annona dioica, Arrabidaea brachypoda, Baccharis dracunculifoli e Bauhinia longifolia. }\end{array}$ \\
\hline Algumas espécies exclusivas do Panga \\
\hline $\begin{array}{l}\text { Acosmium dasycarpum, Alibertia macrophylla, Andira paniculata, Apuleia leiocarpa, Byrsonima basiloba, Cardiopetalum calophyllum, } \\
\text { Connarus suberosus, Davilla elliptica, Didymopanax macrocarpum, Eugenia punicifolia, Eremanthus glomerulatus, Faramea cyanea, } \\
\text { Ficus enormis, Guapira graciliflora, Hancornia speciosa e Hirtella glandulosa. }\end{array}$ \\
\hline Grupo 3 \\
\hline Algumas espécies comuns a todas as áreas \\
\hline Tapirira guianensis, Copaifera langsdorffii e Pera glabrata. \\
\hline Algumas espécies exclusivas da FLONA \\
\hline $\begin{array}{l}\text { Acrocomia aculeata, Alibertia edulis, Allophylus sericeus, Amburana cearensis, Anadenanthera colubrina, Apeiba tibourbou, } \\
\text { Aspidosperma olivaceum, Astronium fraxinifolium, Bauhinia forficata, Callisthene major, Cassia apoucouita, Campomanesia velutina, } \\
\text { Ceiba speciosa, Celtis iguanae, Dilodendron bipinnatum, Eugenia sulcata, Guazuma ulmifolia, Guettarda viburnoides, Hirtella hebeclada } \\
\text { e Machaerium hirtum. }\end{array}$ \\
\hline
\end{tabular}




\section{REFERÊNCIAS}

ARAÚJO, G.M.; HARIDASAN, M. Estrutura fitossociológica de duas matas mesófilas semidecíduas, em Uberlândia, Triângulo Mineiro. Naturalia, v.22, n.1, p.115-129, 1997a.

ARAÚJO, G.M.; NUNES, J.J.N.; ROSA, A.G.; RESENDE, E.R. Estrutura comunitária de vinte áreas de cerrados residuais no município de Uberlândia, MG. Daphne, v.7, n.2, p.7-14, 1997b.

BALDUINO, A.P.C.; SOUZA, A.L.; MEIRA NETO, J.A.A.; SILVA, A.F.; SILVA JUNIOR, M.C. Fitossociologia e análise comparativa da composição florística do cerrado da flora de Paraopeba-MG. Revista Árvore, v.29, n.1, p.25-34, 2005.

CASTRO, A.A.J.F. Composição florísticogeográfica (Brasil) e fitossociológica (Piauí-São Paulo) de amostras de cerrado. Tese (Doutorado em Ciência Florestal) - Universidade Estadual de Campinas, 520p, 1994.

COSTA, A.A.; ARAúJO, G.M. Comparação da vegetação arbórea de Cerradão e de Cerrado na Reserva do Panga, Uberlândia, MG. Acta Botânica Brasílica, v.15, n.1, p.63-72, 2001.

COUTINHO, L.M. O conceito de Cerrado.

Revista Brasileira de Botânica, v.1, n.1, p.17-24, 1978.

DURIGAN, G.; FRANCO, G.A.D.C.; PASTORE, J.A.; AGUIAR, O.T. Regeneração natural da vegetação de Cerrado sob floresta de Eucalyptus citrodora. Revista do Instituto Florestal, v.9, n.1, p.71-85, 1997.

DURIGAN, G.; BACIC, M.C., FRANCO, G.A.D.C.; SIQUEIRA, M.F. Inventário florístico do Cerrado na Estação Ecológica de Assis, SP. Hoehnea, São Paulo, v.26, n.2, p.49-172, 1999.

FELFILI, J.M.; SILVA-JÚNIOR, M.C.; REZENDE, A.V.; MACHADO, J.W.B.; WALTER, B.M.T.; SILVA, P.E.N.; HAY, J. Análise comparativa da florística e fitossociologia da vegetação arbórea do Cerrado sensu stricto na Chapada Pratinha, DF - Brasil. Acta Botanica Brasilica, v.6, n.2, p.27-46, 1993.
FELFILI, J.M.; FILGUEIRAS, T.S.; HARIDASAN, M.; SILVA JR., M.C.; MENDONÇA, R.C.; REZENDE, A.V. Projeto biogeografia do bioma Cerrado: vegetação e solos. Cadernos de Geociências, v.12, n.4, p.75-166, 1994.

GOODLAND, R. Análise ecológica da vegetação de cerrado. In: Goodland, R.; Ferri, M.G. Ecologia do cerrado. Itatiaia, Belo Horizonte e EDUSP, São Paulo, 1969.

HERINGER, E.P.; BARROSO, G.M.; RIZZO, J.A.; RIZZINI, C.T. A Flora do Cerrado. In: SIMPÓSIO SOBRE CERRADO, 6., 1977, Belo Horizonte. Anais... Belo Horizonte: Ed. Itatiaia, p.211-232, 1977.

MANTOVANI, N.; MARTINS, F.R. 1993.

Florística do cerrado na Reserva Biológica de Mogi Guaçu, SP. Acta Botanica Brasilica, v.7, n.1, p.33-60, 1993.

MARTINS, F.R. Atributos de comunidades vegetais. Quid, Teresina, v.9, p.12-17, 1990.

MEIRA NETO, J.A.A. Composição florística e fitossociológica da vegetação de Cerrado Sensu Lato da Estação Ecológica de Santa Bárbara (E.E.S.B.), Município de Águas de Santa Bárbara. Estado de São Paulo. 1991. 98 f. Dissertação (Mestrado em Biologia) - Universidade de Campinas, Campinas, 1991.

MENDONÇA R.C, FELFILI J.M, WALTER B.M.T, SILVA JÚNIOR M.C, REZENDE A.V, FILGUEIRAS T, NOGUEIRA P.E. Flora vascular do cerrado, p. 289-556. In: SANO S.M, ALMEIDA S.P (eds.) Cerrado: Ambiente e Flora. Planaltina, EMBRAPA. 556pp., 1998.

MOBOT. Missouri Botanical Gardem. Disponível em (http://www.mobot.org/W3T/search/vast.html), 2007.

MUELLER-DOMBOIS, D.Y.; ELLENBERG, M. Aims and methods in vegetation ecology. New York: John Wiley e Sons, 547p, 1974.

RATTER, J.A.; DARGIE, T.C.D. Analysis of the floristic composition of 26 cerrado areas in Brazil. Edinburgh Journal of Botany, v.49, n.2, p.235-250, 1992.

R. Árvore, Viçosa-MG, v.32, n.4, p.781-790, 2008 
RATTER, J. A.; P. W. RICHARDS; G. ARGENT; GIFFORD, D. R. Observations on the vegetation of northeastern Mato Grosso 1. The woody vegetation types of the Xavantina-Cachimbo expedition area. Phil. Trans. R. Soc. London (B), n.266, p.449-492, 1973.

RATTER J.A.; RICHARDS, P.W.; ARGENT, G.; GIFFORD, D.R. Observations on the forests of some mesotrophic soils in central Brazil. Revista Brasileira de Botânica, v.1, p.47-58, 1978.

RATTER, J.A.; LEITÃO-FILHO, H.F.; ARGENT, G.; GIBBS, P.E.; SEMIR, J.; SHEPHERD, G.J.; TAMASHIRO, J.Y. 1988. Floristic composition and community structure of a southern cerrado area in Brazil. Notes from the Royal Botanic Garden, Edinburgh, v.45, p.137-51, 1988.

RATTER, J.A.; BRIDGEWATER, S.; ATKINSON, R.; RIBEIRO, J.F. Analysis of the floristic composition of the Brazilian Cerrado vegetation II: comparison of the woody vegetation of 98 areas. Edinburgh Journal of Botany, v.53, n.2, p.153-180, 1996.

RIZZINI, C.T.; HERINGER, E.P. Preliminares acerca das formações vegetais $e$ do reflorestamento no Brasil Central. Rio de Janeiro: Secretaria de Agricultura, 79p, 1962.

RIZZINI, C.T. A flora do Cerrado, análise florística das savanas centrais. In: SIMPÓSIO SOBRE O CERRADO, 1962, São Paulo. Anais... São Paulo: EDUSP, p.125-177, 1963.

RIZZINI, C.T. Aspectos ecológicos da regeneração em algumas plantas do cerrado. In Anais do III Simpósio sobre cerrado (M.G. FERRI, coord.). Editora Edgard Blücher, São Paulo, p.61-64, 1971.

RIZZINI, C.T. Tratado de Fitogeografia do Brasil: aspectos sociológicos e florísticos. HUCITEC/ USP, São Paulo, 1979.

RIBEIRO, J.F.; GONZALES, M.I.; OLIVEIRA, P.E.A.M.; MELO, J.T. Aspectos fenológicos de espécies nativas do Cerrado. In: CONGRESSO NACIONAL DE BOTÂNICA, v.32, 1981, Teresina, PI: Anais... Teresina: Sociedade Botânica do Brasil, p.181-198, 1982.

R. Árvore, Viçosa-MG, v.32, n.4, p.781-790, 2008
RIBEIRO, J.F.; SILVA, J.C.S.; BATMANIAN, G.J. Fitossociologia de tipos fisionômicos de cerrado em planaltina-DF. Revista Brasileira de Botânica, v.8, p.131-142, 1985.

ROYAL BOTANIC GARDENS. Index Kewensis on compact disc - Manual. Oxford University Press, 67p, 1993.

SILVA-JÚNIOR, M.C. Composição florística, estrutura e parâmetros fitossociológicos do Cerrado e sua relação com o solo na Estação Florestal de Experimentação de Paraopeba-MG. Viçosa-MG: UFV, 1984. 130p. Dissertação (Mestrado em Ciência Florestal) - Universidade Federal de Viçosa, 1984.

SHEPERD, G.J. FITOPAC 1. Manual do usuário C 1. Campinas: UNICAMP, Departamento de Botânica, 96p, 2002.

SNEATH, P.H.; SOKAL, R.R. Numerical taxonomy. San Francisco: W.H. Freeman and Company, 573p, 1973.

SOUZA, J.S.; ESPÍRITO-SANTO, F.D.B.; FONTES, M.A.L.; OLIVEIRA-FILHO, A.T.; BOTEZELLI, L. Análise das variações florísticas e estruturais da comunidade arbórea de um fragmento de Floresta Semidecídua às margens do rio Capivari, Lavras, MG. Revista Árvore, v.27, n.2, p.185-206, 2003.

SOUZA, V.C.; LORENZI, H. 2005. BOTÂNICA SISTEMÁTICA: Guia ilustrado para identificação das famílias de Angiospermas da flora brasileira, baseado em APG II. Nova Odessa, SP, Editora: Instituto Plantarum.

THIBAU, C.E.; HEISEKE, D.H.; MOURA, P.V; LAMAS, J.M.; CESAR, R.L. Inventário preliminar expedido na Estação Florestal de Experimentação de Paraopeba em Minas Gerais. Brasil Florestal, v.6, p.34-71, 1975.

THORNWAITE, C.W.; MATHER, J.R. Instructions and tables for computing potential evapotranspiration and water balance.

Centerton: Publication Climat, v.10, n.3, 1957.

WEISER, V.L.; GODOY, S.A.P.. 2001. Florística em um hectare de cerrado stricto sensu na ARIE - cerrado Pé-de-gigante, Santa Rita do Passa Quatro, SP. Acta Botanica Brasílica, v.15, n.2, p. 201-212, 2001. 